- Original Article

\title{
The Correlation between Carotid Intima- Media Thickness and Neutrophil to Lymphocyte Ratio in Prediabetes Patients
}

\author{
Dayoung Lee, Mi-Jeong Park, Mee Young Kim, Jung Jin Cho, Jong Lull Yoon* \\ Department of Family Medicine, Hallym University Dongtan Sacred Heart Hospital, Hwaseong, Korea
}

\begin{abstract}
Background: Prediabetes is a metabolic state between normoglycemia and diabetes and is known to carry a higher risk of developing overt diabetes and cardiovascular disease (CVD). The relative and absolute risks of all-cause mortality, CVD, coronary heart disease, and stroke in prediabetes patients, as well as in diabetic patients, is higher than that in patients with normoglycemia. Carotid intima-media thickness (cIMT) is a method used to stratify CVD risk. In this study, we aimed to determine whether the neutrophil-to-lymphocyte ratio (NLR) correlates with cIMT in prediabetes patients.

Methods: From January 1, 2016, to February 20, 2021, 581 adults their 30s-70s who underwent carotid ultrasonography as part of a comprehensive medical examination at the Dongtan Sacred Heart Hospital were enrolled. Statistical analysis using SPSS presented t-test and chi-square test significance levels into a group with normal cIMT (nIMT; cIMT $<1 \mathrm{~mm}$ ) and a group with thick cIMT (tIMT; cIMT $\geq 1 \mathrm{~mm}$ ). Binary logistic regression analysis was performed to confirm the correlation between NLR and cIMT.

Results: In prediabetic adults, age, hemoglobin Alc (HbAlc), systolic blood pressure, and NLR were significantly higher in the tIMT group than in the nIMT group. In the regression analysis, NLR, age, and HbAlc were significantly correlated with cIMT.

Conclusion: NLR was significantly higher in the tIMT group than in the nIMT group; therefore, NLR may be used to assess CVD risk in prediabetes patients.
\end{abstract}

Keywords: Prediabetic State; Neutrophils; Lymphocytes; Carotid Intima-Media Thickness; Cardiovascular Diseases

Received: March 31, 2021, Revised: July 28, 2021, Accepted: August 27, 2021

${ }^{*}$ Corresponding Author: Jong Lull Yoon https://orcid.org/0000-0001-8931-0256

Tel: +82-31-8086-2390, Fax: +82-31-8086-2709, E-mail: Iull@hallym.or.kr 


\section{INTRODUCTION}

In 2018, the prevalence of diabetes mellitus, commonly known as diabetes, in Korea, was $13.8 \%$ in adults aged 30 years or older and $27.6 \%$ in adults aged 65 or older, increasing with age in both men and women. ${ }^{1)}$ The prevalence of prediabetes was $26.9 \%$ in adults aged 30 years or older and $29.6 \%$ in adults aged 65 or older, and men had a higher prevalence than women by age group. ${ }^{1)}$ Prediabetes is a metabolic state between normoglycemia and diabetes. Similar to diabetes, prediabetes is also a risk factor for cardiovascular disease (CVD). In several studies, a 1\% increase in hemoglobin Alc (HbAlc) within the normal range resulted in increased 10-year cardiovascular mortality, and CVD mortality doubled in the impaired glucose tolerance (IGT) group compared with the normal glucose tolerance (NGT) group. ${ }^{2)}$ In other studies, the incidence of unrecognized myocardial infarction in the prediabetic state was 3 times higher than that in the normal group, and it was proven that patients with diabetes and prediabetes had a poor prognosis 30 days after acute ischemic stroke. ${ }^{2)}$

CVD or stroke is a disease caused by the interaction of several risk factors, including diabetes and genetic predisposition. ${ }^{3)}$ It is the most common cause of death worldwide. ${ }^{4)}$ Carotid intima-media thickness (cIMT) is an early marker for the development and progression of atherosclerosis. ${ }^{5)}$ The pathophysiology of atherosclerosis is attributed to inflammation. ${ }^{6}$ Chronic inflammation in the blood vessel wall is thought to play an important role in the development and progression of atherosclerosis, and leukocytes and platelets are important factors in the occurrence of atherosclerosis. ${ }^{7,8)}$

The neutrophil-to-lymphocyte ratio (NLR) has been studied as a prognostic factor in CVD, lung disease, infection, endocrine disease, and some malignancies. ${ }^{9}$ Although the correlation between NLR and CVD has not been fully established, systemic factors such as inflammation, vascular endothelial cell dysfunction, and oxidative stress are thought to have an effect. ${ }^{10)}$ A higher NLR is expected to lead to faster development and progression of atherosclerosis, and a higher NLR is associated with a higher mortality rate or can be used as a prognostic factor in patients with acute coronary syndrome or percutaneous cardiovascular intervention. ${ }^{8,11,12)}$

The development of atherosclerosis begins with prediabetes. ${ }^{13)}$ The length of the asymptomatic period in diabetes or prediabetes exposes patients to cardiovascular risk factors and increases the incidence of coronary artery disease; therefore, early diagnosis and prevention through appropriate screening is important. ${ }^{14)}$ This study was designed to investigate whether NLR as a predictive marker correlates with cIMT in prediabetes patients.

\section{METHODS}

\section{Study Population}

This retrospective observational study was conducted on patients who underwent a comprehensive medical examination and agreed to share their data for research purposes at the Dongtan Sacred Heart
Hospital from January 1, 2016, to February 20, 2021. In this study, 581 adults aged between 30 and 80 years who underwent carotid ultrasound to evaluate cIMT were enrolled. None of the participants had been diagnosed with diabetes. Participants with malignancy, inflammatory or autoimmune diseases such as rheumatoid arthritis, osteoarthritis, ankylosing spondylitis, Crohn's disease, cardio-cerebrovascular disease, and thyroid disease, which may affect NLR levels, were excluded. Patients who took drugs including antiplatelet agents such as aspirin or anti-thrombotic agents or white blood cell (WBC) count $>10,000 / \mu \mathrm{L}$ were also excluded (Figure 1 ). This study was approved by the institutional review board of the Hallym University Dongtan Sacred Heart Hospital (IRB approval no., HDT 2021-07-015) and omission of informed consents were allowed because of the retrospective design of this study.

\section{Materials and Methods}

The results of all blood tests and anthropometric tests were collected using the results of the comprehensive medical examination at the hospital; and the participants' past history, current medical history (e.g., whether they had been diagnosed with hypertension (HTN), dyslipidemia, diabetes, or other diseases described above, are under treatment, or on medicines) and smoking history were also identified using the interview data obtained during the examination. The diagnosis of metabolic syndrome was defined as having three or more diagnostic criteria using the data on abdominal circumference (AC), fasting blood glucose (FBG), blood pressure, triglyceride (TG), and high-density lipoprotein cholesterol (HDL-C).

All subjects fasted for at least 8 hours before examination, and blood tests were taken from the antecubital vein. The complete blood count (CBC) test was performed using a Sysmex XN-3000 automatic hematology analyzer (Sysmex Corp., Kobe, Japan). The absolute neutrophil count (ANC) and absolute lymphocyte count (ALC) were obtained by multiplying the WBC count by the percentage of neutrophils and lymphocytes, respectively, in the CBC test. The NLR was obtained by dividing the ANC by ALC. Chemistry tests such as FBG, total cholesterol (TC), TG, low-density lipoprotein cholesterol (LDL-C), and HDL-C were analyzed using the Cobas c702 module in the Cobas 8000 modular analyzer series (Roche Diagnostics, Mannheim, Germany). HbAlc was analyzed using the Bio-Rad D-100 hemoglobin testing system or the Variant II turbo analyzer 2.0 (Bio-Rad Laboratories, Hercules, CA, USA). The weight and height were obtained using an InBody 720 (Biospace Co. Ltd., Seoul, Korea), and the AC was measured by a nurse at the institution using a tape measure at the level of the belly button.

Carotid ultrasound was performed by radiology specialists using the IU-22 Philips ultrasound system (Philips Healthcare, Bothell, WA, USA) or GE LOGIQ E10 Ultrasound (GE Healthcare, Wauwatosa, WI, USA), and the cIMT was measured at the common carotid artery. Thickening was defined as a cIMT of $1 \mathrm{~mm}$ or more. Based on these criteria, the patients were divided into two groups: normal cIMT (nIMT $<1 \mathrm{~mm}$ ) and thick cIMT (tIMT $\geq 1 \mathrm{~mm}$ ). 
2016. 1. 1-2021. 2. 20

Enrollment for health check-up
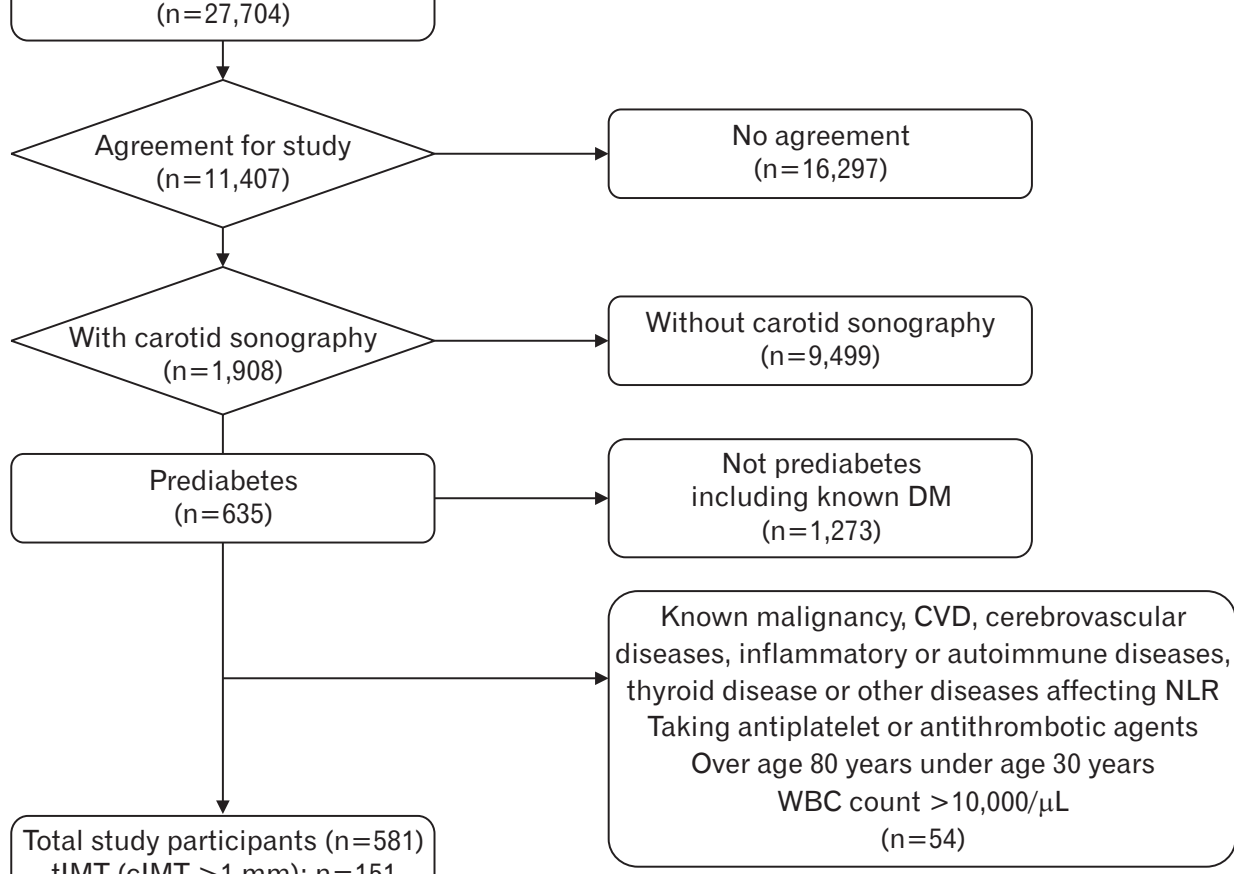

Figure 1. Flowchart of the participants in the study. In total, 581 participants were included. They were divided into two groups according to carotid intima-media thickness (cIMT). Where the cIMT was $1 \mathrm{~mm}$ thick or more, it as categorized as thick cIMT (tIMT). Where the cIMT was less than $1 \mathrm{~mm}$ thick, it was categorized as normal cIMT (nIMT). Of these, 430 and 151 were in the nIMT and tIMT groups, respectively. DM, diabetes mellitus; tIMT (cIMT $\geq 1 \mathrm{~mm}$ ): $\mathrm{n}=151$ nIMT (cIMT <1 mm): n=430 CVD, cardiovascular disease; NLR, neutrophi to lymphocyte ratio; WBC, white blood cell.

Table 1. Baseline characteristics in all participants and comparison between two groups according to cIMT

\begin{tabular}{|c|c|c|c|c|}
\hline \multirow{2}{*}{ Characteristics } & \multirow{2}{*}{ Total $(n=581)$} & \multicolumn{2}{|c|}{ clMT* } & \multirow{2}{*}{ P-value ${ }^{\dagger}$} \\
\hline & & nIMT $(n=430)$ & tIMT $(n=151)$ & \\
\hline Sex & & & & 0.696 \\
\hline Male & $434(74.7)$ & 323 (75.1) & 111 (73.5) & \\
\hline Female & $147(25.3)$ & $107(24.9)$ & $40(26.5)$ & \\
\hline Age (y) & $53.55 \pm 9.29$ & $52.48 \pm 9.29$ & $56.59 \pm 8.62$ & 0.000 \\
\hline 30-39 & 35 (6.0) & $31(7.2)$ & 4 (2.6) & \\
\hline $40-49$ & $169(29.1)$ & $144(33.5)$ & 25 (16.6) & \\
\hline $50-59$ & $233(40.1)$ & $166(38.6)$ & $67(44.4)$ & \\
\hline $60-69$ & $113(19.4)$ & $69(16.0)$ & $44(29.1)$ & \\
\hline 70-79 & $31(5.3)$ & $20(4.7)$ & $11(7.3)$ & \\
\hline Hypertension & & & & 0.097 \\
\hline None & $462(79.5)$ & $349(81.2)$ & $113(74.8)$ & \\
\hline Diagnosed & $119(20.5)$ & 81 (18.8) & 38 (25.2) & \\
\hline Dyslipidemia & & & & 0.413 \\
\hline None & $488(84.0)$ & 358 (83.3) & 130 (86.1) & \\
\hline Diagnosed & $45(7.7)$ & $72(16.7)$ & 21 (13.9) & \\
\hline $\mathrm{MS}^{\ddagger}$ & & & & 0.608 \\
\hline None & $343(59.4)$ & $258(60.0)$ & 87 (57.6) & \\
\hline Diagnosed & $236(40.6)$ & $172(40.0)$ & 64 (42.4) & \\
\hline Smoking & & & & 0.983 \\
\hline None & $257(44.2)$ & $190(43.9)$ & $67(44.4)$ & \\
\hline Current & $148(25.5)$ & $109(25.5)$ & 39 (25.8) & \\
\hline Past & $176(30.3)$ & $131(30.6)$ & 45 (29.8) & \\
\hline
\end{tabular}

Values are presented as number (\%) or mean \pm standard deviation.

cIMT, carotid intima-media thickness; nIMT, normal carotid intima-media thickness; tIMT, thick carotid intima-media thickness; MS, metabolic syndrome.

${ }^{*}$ CIMT less than $1 \mathrm{~mm}$ defined as nIMT and cIMT $1 \mathrm{~mm}$ or over defined as tIMT. The P-value of all variables except mean of age was presented using chi-square analysis. The mean and standard deviation value of age was analyzed using t-test. " The diagnosis of MS was defined as having three or more diagnostic criteria including abdominal circumference, fasting blood glucose, blood pressure, triglyceride, and high-density lipoprotein cholesterol. 


\section{The Definition of Prediabetes}

Prediabetes can be mainly divided into impaired fasting glucose (IFG) and IGT, and the criteria for HbAlc have been added. ${ }^{15,16)}$ IFG is defined as FBG between 100 and $125 \mathrm{mg} / \mathrm{dL}$, IGT is defined as PP2 between 140 and $199 \mathrm{mg} / \mathrm{dL} 2$ hours after the oral glucose tolerance test (OGTT), and HbAlc between 5.7\% and 6.4\%. ${ }^{15)}$ Because there were insufficient data on OGTT, in this study, prediabetes was defined using cases of IFG between 100 and $125 \mathrm{mg} / \mathrm{dL}$ or HbAlc between $5.7 \%$ and $6.4 \%$.

\section{Statistical Analysis}

Continuous variables, such as blood tests, were presented as mean and standard deviation. The normality assumption was evaluated using the Shapiro-Wilk test, and the homogeneity of variance was evaluated using Levene's test. Variables that were not satisfied with the normality assumption were log-transformed, a t-test was conducted, and the P-value was subsequently presented. Categorical variables such as gender, smoking history, and disease history were presented as numbers with percentiles and compared by performing a chi-square test. Binary logistic regression was performed to confirm the correlation between cIMT and NLR by adjusting for sex, age, high-sensitivity C-reactive protein (hs-CRP), FBG, HbAlc, HDL-C, AC, HTN or dyslipidemia diagnosis, and current smoking status. The odds ratios (ORs) of all variables were presented in three cases (i.e., unadjusted, model I, and model II) using logistic regression analysis; age and sex were adjusted in model I, and all variables were adjusted in model II. All statistical analyses were performed using IBM SPSS Statistics for Windows ver. 26.0 (IBM Corp., Armonk, NY, USA). All significance tests were 2-tailed, and values of $\mathrm{P}<0.05$ were considered significant.

\section{RESULTS}

\section{Baseline Characteristics}

The baseline characteristics and blood test results of the study participants are shown in Tables 1 and 2. Among the 581 study participants, $434(74.7 \%)$ were male and 147 (25.3\%) were female, with an average age of $53.55 \pm 9.29$ years. There were 151 participants with a tIMT and 430 participants with nIMT. There were 111 male participants in the nIMT group and 323 male participants in the tIMT group; whilst there were 40 and 107 female participants in the two groups, respectively.

Regarding the characteristics of the subjects according to cIMT, the average age of the IIMT group was 56.59 \pm 8.62 years, which was higher than the $52.48 \pm 9.29$ years of the nIMT group $(\mathrm{P}<0.001)$. For men, the average age of the IIMT group was $55.62 \pm 8.85$ years, which was higher than the $51.11 \pm 8.88$ years of the nIMT group. For women, the average age of each group was $59.28 \pm 7.40$ years and $56.61 \pm 9.31$ years, respectively. NLR was $1.89 \pm 0.90$ in the tIMT group and $1.71 \pm 0.69$ in the nIMT group. NLR was significantly higher in the tIMT group than in the nIMT group $(\mathrm{P}=0.018)$. For men, NLR was $1.91 \pm 0.88$ in the tIMT group and $1.72 \pm 0.70$ in the nIMT group. For women, the NLR was $1.84 \pm 0.97$ and $1.65 \pm 0.63$ in each group, respectively. FBG and HbAlc were $105.54 \pm 11.05 \mathrm{mg} / \mathrm{dL}$ and $5.85 \% \pm 0.35 \%$, respectively, in the tIMT group, and $104.30 \pm 10.45 \mathrm{mg} / \mathrm{dL}$ and $5.71 \% \pm 0.34 \%$ in the nIMT group. HbAlc was significantly higher in the tIMT group than in the nIMT group

Table 2. Laboratory results in all participants and comparison between two groups according to cIMT

\begin{tabular}{|c|c|c|c|c|}
\hline \multirow{2}{*}{ Variable } & \multirow{2}{*}{ Total $(n=581)$} & \multicolumn{2}{|c|}{$\mathrm{CIMT}^{*}$} & \multirow{2}{*}{ P-value } \\
\hline & & nIMT (n=430) & tIMT $(n=151)$ & \\
\hline White blood cell $\left(\times 10^{3} / \mu \mathrm{L}\right)$ & $5.63 \pm 1.39$ & $5.59 \pm 1.42$ & $5.75 \pm 1.32$ & 0.145 \\
\hline Absolute neutrophil count $(/ \mu \mathrm{L})$ & $3,128.20 \pm 1,042.63$ & $3,078.11 \pm 1,022.66$ & $3,270.85 \pm 1,088.39$ & 0.057 \\
\hline Absolute lymphocyte count $(/ \mu \mathrm{L})$ & $1,886.97 \pm 523.72$ & $1,901.64 \pm 536.50$ & $1,845.18 \pm 484.74$ & 0.357 \\
\hline Neutrophil to lymphocyte ratio & $1.75 \pm 0.75$ & $1.71 \pm 0.69$ & $1.89 \pm 0.90$ & 0.018 \\
\hline Hemoglobin (g/dL) & $14.73 \pm 1.30$ & $14.74 \pm 1.33$ & $14.69 \pm 1.21$ & 0.741 \\
\hline Platelet $\left(\times 10^{3} / \mu \mathrm{L}\right)$ & $244.76 \pm 51.38$ & $245.60 \pm 51.99$ & $242.38 \pm 49.71$ & 0.488 \\
\hline Fasting blood glucose (mg/dL) & $104.63 \pm 10.62$ & $104.30 \pm 10.45$ & $105.54 \pm 11.05$ & 0.230 \\
\hline Total cholesterol (mg/dL) & $196.65 \pm 39.67$ & $196.39 \pm 39.23$ & $197.37 \pm 41.02$ & 0.907 \\
\hline Triglyceride (mg/dL) & $153.74 \pm 99.20$ & $151.78 \pm 95.62$ & $159.33 \pm 108.88$ & 0.288 \\
\hline High-density lipoprotein cholesterol (mg/dL) & $52.30 \pm 13.34$ & $52.56 \pm 13.12$ & $51.56 \pm 13.94$ & 0.340 \\
\hline Low-density lipoprotein cholesterol (mg/dL) & $133.84 \pm 37.32$ & $132.72 \pm 36.73$ & $137.02 \pm 38.90$ & 0.329 \\
\hline Hemoglobin A1c (\%) & $5.74 \pm 0.34$ & $5.71 \pm 0.34$ & $5.85 \pm 0.35$ & 0.000 \\
\hline Erythrocyte sedimentation rate (mm/h) & $12.86 \pm 10.29$ & $12.64 \pm 10.08$ & $13.47 \pm 10.88$ & 0.209 \\
\hline High-sensitivity C-reactive protein (mg/L) & $1.23 \pm 2.40$ & $1.22 \pm 2.44$ & $1.28 \pm 2.28$ & 0.218 \\
\hline Height $(\mathrm{cm})$ & $167.54 \pm 8.84$ & $167.93 \pm 8.75$ & $166.43 \pm 9.02$ & 0.072 \\
\hline Weight (kg) & $72.07 \pm 13.30$ & $72.48 \pm 13.45$ & $70.93 \pm 12.83$ & 0.229 \\
\hline Body mass index $\left(\mathrm{kg} / \mathrm{m}^{2}\right)$ & $25.54 \pm 3.44$ & $25.57 \pm 3.49$ & $25.47 \pm 3.31$ & 0.821 \\
\hline Abdomen circumference (cm) & $87.38 \pm 9.26$ & $87.36 \pm 9.07$ & $87.44 \pm 9.82$ & 0.999 \\
\hline Systolic blood pressure (mm Hg) & $121.78 \pm 14.40$ & $120.65 \pm 13.50$ & $124.99 \pm 16.31$ & 0.002 \\
\hline Diastolic blood pressure (mm Hg) & $73.87 \pm 11.07$ & $73.73 \pm 10.74$ & $74.26 \pm 11.99$ & 0.700 \\
\hline
\end{tabular}

Values are presented as mean \pm standard deviation.

CIMT, carotid intima-media thickness; nIMT, normal carotid intima-media thickness; tIMT, thick carotid intima-media thickness.

${ }^{*}$ CIMT less than $1 \mathrm{~mm}$ defined as nIMT and cIMT $1 \mathrm{~mm}$ or over defined as tIMT. The P-value of all variables was presented using t-test. 
$(\mathrm{P}<0.001)$, whereas FBG levels were not significantly different between the two groups $(\mathrm{P}=0.230)$. For men, HbAlc was $5.85 \% \pm 0.37 \%$ in the tIMT group and $5.69 \% \pm 0.34 \%$ in the nIMT group. For women, HbAlc was $5.84 \% \pm 0.27 \%$ and $5.78 \% \pm 0.31 \%$ in each group respectively. In addition, systolic blood pressure (SBP) was $124.99 \pm 16.31 \mathrm{~mm} \mathrm{Hg}$ and $120.65 \pm 13.50 \mathrm{~mm} \mathrm{Hg}$, respectively, which showed higher SBP in the tIMT group than nIMT group $(\mathrm{P}=0.002)$. For men, SBP was $125.39 \pm 12.97 \mathrm{~mm}$ Hg in tIMT group and $121.29 \pm 12.68 \mathrm{~mm}$ Hg in nIMT group. For women, SBP was $123.88 \pm 23.40 \mathrm{~mm} \mathrm{Hg}$ and $118.71 \pm 15.64$ $\mathrm{mm} \mathrm{Hg}$ in each group, respectively. There were no statistically significant differences in the other variables.

\section{The Correlation between cIMT Thickening and NLR in Binary Logistic Regression Analysis}

Tables 1 and 2 show the differences between the two groups according to the cIMT. Variables that could affect cIMT in previous studies were sex, age, hs-CRP, FBG, HbAlc, HDL-C, AC, HTN, dyslipidemia, current smoking status. ${ }^{17,18)}$ However, some of these factors were not significantly correlated with cIMT in this study. Binary logistic regression analysis with NLR was performed on the variables listed above to adjust for them (Table 3).

In the regression analysis, there was a statistically significant correlation in age, NLR, and HbAlc according to the thickness of cIMT. The unadjusted OR of NLR was 1.354 (95\% confidence interval [CI], 1.071$1.710)$ and 1.368 (95\% CI, 1.078-1.737) after adjusting for age and sex ( $\mathrm{P}=0.011$ and $\mathrm{P}=0.010$, respectively). In model II, subjects in the tIMT group were older and had higher HbAlc levels than those in the nIMT group (OR, 1.046; 95\% CI, 1.022-1.070; $\mathrm{P}<0.001$; OR, 2.426; 95\% CI,
1.289-4.567; $\mathrm{P}=0.006$, respectively), and NLR was higher in the tIMT group (OR,1.334; 95\% CI, 1.038-1.714; $\mathrm{P}=0.024$ ).

\section{DISCUSSION}

In this retrospective study, we evaluated whether there was a significant correlation between cIMT and NLR in adults who were in a prediabetic state but had not been previously diagnosed with diabetes. NLR was significantly higher in the tIMT group than in the nIMT group, and age, HbAlc, and SBP showed the same association. In the logistic regression analysis, NLR was independently correlated with cIMT after adjusting for other variables, although the OR of HbAlc was higher than that of NLR. Concerning HbAlc, the results of this study were consistent with those of several other studies that showed that cIMT was correlated with HbAlc in prediabetes and diabetes subjects. ${ }^{13,19,20)}$ Various studies and meta-analyses revealed that age, HTN, SBP, smoking history, insulin resistance, and metabolic syndrome affect cIMT, and TC or LDL-C and HDL-C, hs-CRP, fibrinogen, LOX-1, and adiponectin were found to be related to cIMT. ${ }^{17)}$ However, there was no statistically significant difference associated to some variables in this study. This may be attributed to the characteristics of the study participants, prediabetes, or the limited sample size. Furthermore, certain variables showed the heterogeneity of the association with cIMT $^{16)}$ and we did not exclude participants taking lipid-lowering agents; alternatively, we adjusted for the factor by including participants diagnosed with dyslipidemia, in the logistic regression analysis.

It is known that the risk of atherosclerotic CVD is increased 2 to 4 times and cardiovascular mortality or morbidity is higher in patients

Table 3. Binary logistic regression analysis with variables affecting cIMT

\begin{tabular}{|c|c|c|c|c|}
\hline Variable & B & SE & $\mathrm{OR}(95 \% \mathrm{Cl})$ & P-value \\
\hline \multicolumn{5}{|l|}{ Unadjusted } \\
\hline$N L R^{*}$ & 0.303 & 0.119 & $1.354(1.071-1.710)$ & 0.011 \\
\hline \multicolumn{5}{|l|}{ Model I } \\
\hline Female & -0.145 & 0.228 & $0.865(0.554-1.351)$ & 0.524 \\
\hline $\mathrm{Age}^{*}$ & 0.051 & 0.011 & $1.052(1.030-1.075)$ & 0.000 \\
\hline$N L R^{*}$ & 0.313 & 0.122 & $1.368(1.078-1.737)$ & 0.010 \\
\hline \multicolumn{5}{|l|}{ Model II } \\
\hline Female & -0.120 & 0.265 & $0.887(0.528-1.490)$ & 0.650 \\
\hline $\mathrm{Age}^{\star}$ & 0.045 & 0.012 & $1.046(1.022-1.070)$ & 0.000 \\
\hline$N L R^{\star}$ & 0.288 & 0.128 & $1.334(1.038-1.714)$ & 0.024 \\
\hline $\mathrm{Hs}-\mathrm{CRP}(\mathrm{mg} / \mathrm{L})^{*}$ & -0.005 & 0.040 & $0.995(0.919-1.077)$ & 0.898 \\
\hline $\mathrm{FBG}(\mathrm{mg} / \mathrm{dL})^{*}$ & 0.006 & 0.009 & $1.006(0.987-1.024)$ & 0.545 \\
\hline $\mathrm{HbA1c}(\%)^{*}$ & 0.886 & 0.323 & $2.426(1.289-4.567)$ & 0.006 \\
\hline $\mathrm{HDL}-\mathrm{C}(\mathrm{mg} / \mathrm{dL})^{*}$ & -0.004 & 0.008 & $0.996(0.980-1.012)$ & 0.595 \\
\hline $\mathrm{AC}(\mathrm{cm})^{\star}$ & -0.004 & 0.013 & $0.996(0.972-1.021)$ & 0.737 \\
\hline HTN diagnosed & 0.276 & 0.243 & 1.318 (0.819-2.123) & 0.256 \\
\hline DL diagnosed & -0.320 & 0.286 & $0.726(0.414-1.272)$ & 0.263 \\
\hline Current smoker & 0.084 & 0.245 & $1.088(0.673-1.759)$ & 0.732 \\
\hline
\end{tabular}

Dependent variable: cIMT (normal cIMT is reference group). Model l: adjusted for sex and age; model II: adjusted for sex, age, hs-CRP, FBG, HbA1c, HDL-C, AC, history of HTN and DL, and smoking status.

CIMT, carotid intima-media thickness; SE, standard error; OR, odds ratio; Cl, confidence interval; NLR, neutrophil to lymphocyte ratio; hs-CRP, high-sensitivity C-reactive protein; FBG, fasting blood glucose; HbA1c, hemoglobin A1c; HDL-C, high-density lipoprotein cholesterol; AC, abdomen circumference; HTN, hypertension; DL, dyslipidemia.

${ }^{*}$ Continuous variables. 
with type 2 diabetes mellitus than in those without diabetes. ${ }^{16)}$ According to a recent meta-analysis, the relative risk of all-cause mortality, composite CVD, coronary heart disease (CHD), and stroke were higher in prediabetes patients than in those with normoglycemia, and the absolute risk was also higher in prediabetes patients. ${ }^{21)}$ Even in patients with atherosclerotic CVD, patients with prediabetes were at a higher risk of all-cause mortality, composite CVD, and CHD than those with normoglycemia. ${ }^{21)}$ In many large-cohort studies that have studied the relationship between cIMT and the risk of developing CVD and stroke, it has been found that cIMT helps stratify the risk of CVD. ${ }^{17}$ In a systematic review, cIMT was found to be thicker in patients with diabetes or IGT than in the control group. ${ }^{18)}$

Li et al. ${ }^{19)}$ found that a high NLR was correlated with thick cIMT in diabetic patients and that NLR could be used as an independent risk factor for the development of asymptomatic atherosclerosis. Few studies have confirmed the relationship between NLR and cIMT in prediabetes. According to a previous Korean study, cIMT was significantly related to age and HbAlc in men with a HbAlc lower than 6.5\%, whilst in women, there was a significant correlation with age; however, NLR was not evaluated in that study. ${ }^{20)}$ The strength of this study is that it revealed the association between NLR and cIMT in prediabetes patients, rather than diabetes patients, which is an understudied area. The mechanism by which NLR influences cIMT is not yet known. Chronic inflammation is known to play an important role in diabetes complications as well as in the development and progression of diabetes and prediabetes. ${ }^{2,22)}$ Several studies have shown increased levels of proinflammatory cytokines in prediabetic and diabetic patients. ${ }^{2,23,24)}$ It has been found that the hyperglycemic state continuously activates neutrophils, ${ }^{25)}$ and activated neutrophils attach to vascular endothelial cells, causing damage to vascular endothelial cells. ${ }^{26)}$ Lymphocytopenia is also considered an inflammatory marker, and when corticosteroid levels increase in response to stress, inflammatory reactions and lymphocyte apoptosis occur, leading to lymphocytopenia. ${ }^{27)}$

This study has some limitations. First, it may be difficult to generalize to the public population due to the sample size and the fact that the study data were obtained from a single medical institution. Second, there may be limitations in identifying a participant's entire medical history through questionnaires and interviews, which could have led to missing information. Third, IGT could not be included because there were insufficient OGTT records in the comprehensive examination. Fourth, the linear relationship could not be confirmed because some cIMT data could not be quantified. Fifth, except for the blood tests analyzed in this study, we did not exclude cases with abnormalities in liver, renal function or thyroid function tests (TFTs) that could cause chronic inflammation, thereby affecting cIMT results. in some studies, there was a significant positive correlation between alanine aminotransferase, $\gamma$-glutamyl transpeptidase, and cIMT and a negative correlation between eGFR and cIMT. ${ }^{28,29)}$ There was a significant difference in cIMT according to TFT, and cIMT was higher in subclinical hypothyroidism than in the euthyroid state. ${ }^{30)}$

In this study, the NLR was found to be higher in prediabetic adults with thick cIMT. In the logistic regression analysis, NLR showed an equal correlation with cIMT to an independent factor. Compared to other blood tests, NLR is a cost-effective means of evaluating CVD in primary care, and it is expected that NLR will be used as an indicator for predicting the risk of CVD in patients with prediabetes. Therefore, further research including large-scale prospective cohort studies are required to confirm the results of our study.

\section{CONFLICT OF INTEREST}

No potential conflict of interest relevant to this article was reported.

\section{ORCID}

Dayoung Lee: https://orcid.org/0000-0002-5811-2852

Mi-Jeong Park: https://orcid.org/0000-0003-1664-4452

Mee Young Kim: https://orcid.org/0000-0002-1724-2175

Jung Jin Cho: https://orcid.org/0000-0001-8871-8858

Jong Lull Yoon: https://orcid.org/0000-0001-8931-0256

\section{REFERENCES}

1. Jung CH, Son JW, Kang S, Kim WJ, Kim HS, Kim HS, et al. Diabetes fact sheets in Korea, 2020: an appraisal of current status. Diabetes Metab J 2021;45:1-10.

2. Brannick B, Dagogo-Jack S. Prediabetes and cardiovascular disease: pathophysiology and interventions for prevention and risk reduction. Endocrinol Metab Clin North Am 2018;47:33-50.

3. Piepoli MF, Hoes AW, Agewall S, Albus C, Brotons C, Catapano AL, et al. 2016 European Guidelines on cardiovascular disease prevention in clinical practice: the sixth Joint Task Force of the European Society of Cardiology and Other Societies on Cardiovascular Disease Prevention in Clinical Practice (constituted by representatives of 10 societies and by invited experts) developed with the special contribution of the European Association for Cardiovascular Prevention \& Rehabilitation (EACPR). Eur Heart J 2016;37:2315-81.

4. Mathers CD, Boerma T, Ma Fat D. Global and regional causes of death. Br Med Bull 2009;92:7-32.

5. Burke GL, Evans GW, Riley WA, Sharrett AR, Howard G, Barnes RW, et al. Arterial wall thickness is associated with prevalent cardiovascular disease in middle-aged adults: the Atherosclerosis Risk in Communities (ARIC) Study. Stroke 1995;26:386-91.

6. Cybulsky MI, Gimbrone MA Jr. Endothelial expression of a mononuclear leukocyte adhesion molecule during atherogenesis. Science 1991;251:788-91.

7. Kim JG, Lee MJ, Kim YH, Kim K, Lee SY, Kim JW, et al. How well can matrix of ferritin and neutrophil to lymphocyte ratio predict metabolic syndrome in Korean adults? Korean J Fam Pract 2020;10:256-61.

8. Thyagaraj, Mohan M, Sreedevi. A study of platelet to lymphocyte ratio in patients with metabolic syndrome. Int J Adv Med 2020;7:737-40.

9. Tulgar YK, Cakar S, Tulgar S, Dalkilic O, Cakiroglu B, Uyanik BS. The effect of smoking on neutrophil/lymphocyte and platelet/lymphocyte ratio and platelet indices: a retrospective study. Eur Rev Med Pharma- 
col Sci 2016;20:3112-8.

10. Demirkol S, Balta S, Unlu M, Arslan Z, Cakar M, Kucuk U, et al. Neutrophils/lymphocytes ratio in patients with cardiac syndrome $\mathrm{X}$ and its association with carotid intima-media thickness. Clin Appl Thromb Hemost 2014;20:250-5.

11. Tamhane UU, Aneja S, Montgomery D, Rogers EK, Eagle KA, Gurm HS. Association between admission neutrophil to lymphocyte ratio and outcomes in patients with acute coronary syndrome. Am J Cardiol 2008;102:653-7.

12. Duffy BK, Gurm HS, Rajagopal V, Gupta R, Ellis SG, Bhatt DL. Usefulness of an elevated neutrophil to lymphocyte ratio in predicting longterm mortality after percutaneous coronary intervention. Am J Cardiol 2006;97:993-6.

13. Scicali R, Giral P, Gallo A, Di Pino A, Rabuazzo AM, Purrello F, et al. HbAlc increase is associated with higher coronary and peripheral atherosclerotic burden in non diabetic patients. Atherosclerosis 2016;255:102-8.

14. Cubbon R, Kahn M, Kearney MT. Secondary prevention of cardiovascular disease in type 2 diabetes and prediabetes: a cardiologist's perspective. Int J Clin Pract 2008;62:287-99.

15. American Diabetes Association. Diagnosis and classification of diabetes mellitus. Diabetes Care 2014;37 Suppl 1:S81-90.

16. Shah AD, Langenberg C, Rapsomaniki E, Denaxas S, Pujades-Rodriguez M, Gale CP, et al. Type 2 diabetes and incidence of cardiovascular diseases: a cohort study in 1.9 million people. Lancet Diabetes Endocrinol 2015;3:105-13.

17. Nezu T, Hosomi N, Aoki S, Matsumoto M. Carotid intima-media thickness for atherosclerosis. J Atheroscler Thromb 2016;23:18-31.

18. Touboul PJ, Hennerici MG, Meairs S, Adams H, Amarenco P, Bornstein $\mathrm{N}$, et al. Mannheim carotid intima-media thickness and plaque consensus (2004-2006-2011): an update on behalf of the advisory board of the $3 \mathrm{rd}, 4$ th and 5th watching the risk symposia, at the 13th, 15th and 20th European Stroke Conferences, Mannheim, Germany, 2004, Brussels, Belgium, 2006, and Hamburg, Germany, 2011. Cerebrovasc Dis 2012;34:290-6.

19. Li X, Shen J, Lu Z, Chen M, Fang X, Wang G. High neutrophil-to-lymphocyte ratio is associated with increased carotid artery intima-media thickness in type 2 diabetes. J Diabetes Investig 2017;8:101-7.

20. Ryu Y, Park Y, Ye BJ. The correlation between hemoglobin Alc and common carotid artery intima medial thickness of both genders with less than $6.5 \%$ HbAlc based on ultrasound measurement. Korean J Health Promot 2015;15:47-53.

21. Cai X, Zhang Y, Li M, Wu JH, Mai L, Li J, et al. Association between prediabetes and risk of all cause mortality and cardiovascular disease: updated meta-analysis. BMJ 2020;370:m2297.

22. Mertoglu C, Gunay M. Neutrophil-lymphocyte ratio and platelet-lymphocyte ratio as useful predictive markers of prediabetes and diabetes mellitus. Diabetes Metab Syndr 2017;11 Suppl 1:S127-31.

23. De Rooij SR, Nijpels G, Nilsson PM, Nolan JJ, Gabriel R, BobbioniHarsch E, et al. Low-grade chronic inflammation in the relationship between insulin sensitivity and cardiovascular disease (RISC) population: associations with insulin resistance and cardiometabolic risk profile. Diabetes Care 2009;32:1295-301.

24. Garcia C, Feve B, Ferre P, Halimi S, Baizri H, Bordier L, et al. Diabetes and inflammation: fundamental aspects and clinical implications. Diabetes Metab 2010;36:327-38.

25. Geerlings SE, Hoepelman AI. Immune dysfunction in patients with diabetes mellitus (DM). FEMS Immunol Med Microbiol 1999;26:259-65.

26. Van Oostrom AJ, van Wijk JP, Sijmonsma TP, Rabelink TJ, Castro Cabezas M. Increased expression of activation markers on monocytes and neutrophils in type 2 diabetes. Neth J Med 2004;62:320-5.

27. Hotchkiss RS, Karl IE. The pathophysiology and treatment of sepsis. N Engl J Med 2003;348:138-50.

28. Kang JH, Cho KI, Kim SM, Lee JY, Kim JJ, Goo JJ, et al. Relationship between nonalcoholic fatty liver disease and carotid artery atherosclerosis beyond metabolic disorders in non-diabetic patients. J Cardiovasc Ultrasound 2012;20:126-33.

29. Wu Y, Hou J, Li J, Luo Y, Wu S. Correlation between carotid intima-media thickness and early-stage chronic kidney disease: results from asymptomatic polyvascular abnormalities in community study. J Stroke Cerebrovasc Dis 2016;25:259-65.

30. Volzke H, Robinson DM, Schminke U, Ludemann J, Rettig R, Felix SB, et al. Thyroid function and carotid wall thickness. J Clin Endocrinol Metab 2004;89:2145-9. 